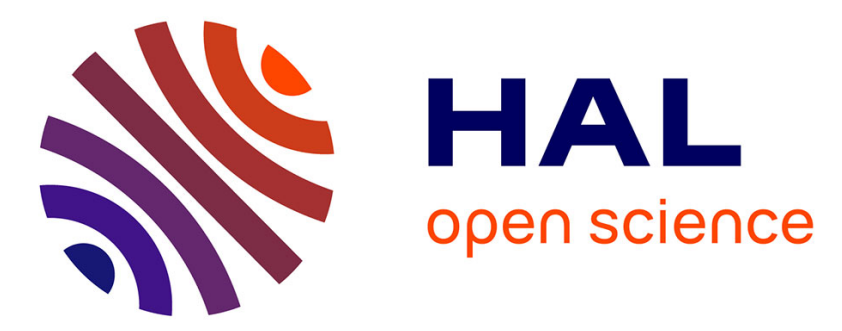

\title{
Qualitative and quantitative DEM analysis of cohesive granular material behaviour in FT4 shear tester
}

Haithem Louati, Xavier Bednarek, Sylvain Martin, Abibatou Ndiaye, Olivier Bonnefoy

\section{- To cite this version:}

Haithem Louati, Xavier Bednarek, Sylvain Martin, Abibatou Ndiaye, Olivier Bonnefoy. Qualitative and quantitative DEM analysis of cohesive granular material behaviour in FT4 shear tester. Chemical Engineering Research and Design, 2019, 148, pp.155 à 163. 10.1016/j.cherd.2019.05.059 . hal-02163782

\section{HAL Id: hal-02163782 \\ https://hal.science/hal-02163782}

Submitted on 24 Jun 2019

HAL is a multi-disciplinary open access archive for the deposit and dissemination of scientific research documents, whether they are published or not. The documents may come from teaching and research institutions in France or abroad, or from public or private research centers.
L'archive ouverte pluridisciplinaire HAL, est destinée au dépôt et à la diffusion de documents scientifiques de niveau recherche, publiés ou non, émanant des établissements d'enseignement et de recherche français ou étrangers, des laboratoires publics ou privés. 


\title{
Qualitative and Quantitative DEM analysis of cohesive granular material behaviour in FT4 shear tester
}

\author{
Haithem Louati ${ }^{1}$, Xavier Bednarek ${ }^{1}$, Sylvain Martin ${ }^{1 *}$, Abibatou Ndiaye ${ }^{2}$ \& Olivier Bonnefoy ${ }^{1}$ \\ ${ }^{1}$ Centre SPIN, Ecole Des Mines de Saint-Etienne, 158 Cours Fauriel, F-42023 Saint-Etienne Cedex 2, France \\ ${ }^{2}$ Orano, B.P. 93124, 30203 Bagnols sur Cèze Cedex, France \\ *Corresponding author contact, Tel: +33 (0)4 774266 94, email address: sylvain.martin@emse.fr (S. Martin)
}

\begin{abstract}
The macroscopic behaviour of cohesive granular material in the FT4 shear tester is studied using the discrete element method (DEM). The shear test is simulated faithfully to the experimental procedure (filling, compaction, pre-shearing and shearing). The angle of internal friction and the apparent bulk cohesion are the macroscopic properties analysed as a result of the variation of the microscopic parameters: the sliding friction coefficient and the adhesive surface energy. The simplified JKR model was used to account for the cohesive contact between spheres. The results of the shear test show that the adhesive forces influence the dilatancy of the granular bed and the incipient failure point. In general, the shear stress increases with the adhesive energy. The sliding friction coefficient and the adhesive energy affect the Yield locus and therefore the angle of internal friction and the apparent cohesion. Two correlations were established between the angle of internal friction and sliding friction coefficient and between cohesion and adhesive energy. The effect of the initial consolidation on the shear test results is also discussed.
\end{abstract}

Keywords: FT4 shear test, DEM, JKR model, Apparent cohesion, Angle of internal friction, Consolidation

\section{Introduction}

Shear testing methods have long been used to characterise the flow behaviour of granular materials. They have been successful in process equipment's design, particularly for bulk materials storage vessels (bins, silos, and hoppers) (Jenike, 1967; Schwedes and Schulze, 1990) as well as for comparing and classifying the flowability of powders. The results obtained from these methods describe the bulk behaviour of the granular materials at critical condition (i.e. the maximum resistance of the granular bed under shearing, called also failure condition) and allow quantifying properties such as shear strength and shearing strain. Difficulties come after to interpret the results and to find the relationship between the individual particle properties and the bulk behaviour. The properties of the material have a very large effect on the shear test results such as the yield stress, apparent cohesion, bulk density, etc. However, since the shear strength is statedependent, the effect of the shear cell type and the shear test operational procedure should not be neglected. A comparison study between different direct shear test devices: Jenike translational shear tester (Jenike, 1964), Schulze ring shear tester (Schulze, 2008), FT4 powder rheometer (Freeman, 2007) and Brookfield powder flow tester (Berry et al., 2015), show a deviation between the results, e.g. the Brookfield and the FT4 show a lower shear stress in comparison to the rest of devices (Leturia et al., 2014; Koynov et al., 2015; Shi et al., 2018). Each device has an operational range of normal stress where results are more accurate, e.g. Jenike for low normal stresses ( $0.5 \mathrm{kPa}-10 \mathrm{kPa})$, FT4 for low and intermediate stresses (1 $\mathrm{kPa}-20 \mathrm{kPa}$ ), and Schulze for low, intermediate and high stresses ( $0.5 \mathrm{kPa}-50 \mathrm{kPa}$ ) (Shi et al., 2018). 
Regardless the shear test types, the consolidation state has a significant effect on the shear test results (Schwedes, 2002; Vasilenko, 2013; Koynov et al, 2015). After compaction, the granular material keeps memory of a consolidation state history, which is maintained even during the steady state flow (Schwedes, 2002). The consolidation stress influences the density of the granular material and hence the measurement of the shear stress. Cohesive granular materials are more sensitive to the consolidation state due to their compressible feature, which is investigated in this paper.

The Discrete Element Method (DEM) is a numerical simulation technique to study the dynamic behaviour of granular systems based on the interactions between neighbour particles. A small time-step, based on the Rayleigh wave speed (Cundall and Strack 1979; Ning and Ghadiri, 2006), is used to update the positions of particles, the created and lost contacts and the associated contact forces. This method has been used to study the flow behaviour of powders in different processes in order to provide a microscopic characterisation of powder, optimisation of process or equipment's design. DEM relies on simple models and simple representation of real materials (e.g., using spheres instead of complicated geometry of particles and reducing the number of these spheres) in order to avoid an exhaustive simulation. Thus, a calibration of particles parameters is necessary in order to provide accurate results. Some processes, such as some mixers, are complicated to be used for calibration and comparison between simulation and experiment. The lack of experimental data and the risk of disturbance during sampling can make the calibration inaccurate. Some shear testers have been used for calibration and have given accurate results such as the triaxial compression test for excavator digging in soil (Obermayr et al., 2014). Shear-box and compression tests were used to calibrate friction coefficient and particle stiffness, where the validation was made by modelling of a blade moving through granular material (Coetzee \& Els, 2009). Schulze ring shear test was used to calibrate Young's modulus, static and rolling friction coefficients in order to model agitated mixers (Simons et al, 2015).

Nowadays, the FT4 powder shear cell is commonly used for powder characterisation both in industry and academia. The major advantage of using FT4 shear test is the fact that the different operational steps are completely automated, which reduces the margin of error. The DEM was used to study annular shear cell such as Schulze device but, for the best of our knowledge, there is no record of shear test DEM simulation using FT4 in the literature. A previous study (Bednarek et al., 2017) investigated the calibration of DEM parameters on FT4 shear test using Kriging method. In this work, we aim to provide information about the FT4 shear-test from DEM point of view and contribute to understanding the shear response of cohesive granular materials and to provide quantitative analysis for calibration. This paper is structured as follows: first, we investigate the shear stress response and dilatant behaviour of non-cohesive and cohesive granular materials. Second, we focus on the effect of the consolidation on the DEM shear test simulation. Then, we discuss how the microscopic DEM parameters (sliding friction coefficient, adhesive energy) influence the macroscopic properties (bulk cohesion, angle of internal friction, dilatancy).

\section{Materials and methods}

\subsection{Shear testing by FT4}

In order to characterise the macroscopic properties of powder flow and quantify the bulk cohesion and the angle of internal friction, the FT4 shear-test was simulated using the discrete element method. The test allows measuring the shear stress response, $\tau$, at a normal stress, $\sigma$, applied to the granular bed (see Fig.2). The shear cell is composed of a cylindrical vessel with two compartments. After pouring the granular material in the chamber, the upper compartment of the vessel rotates and hence removes the extra volume of the sample. This allows reducing the effect of imprecision due to preparation of samples in case of multiples measurements. Then the experimental operational procedure consists of four main steps (see Fig. 1) (ASTM D7891-15, 2015). 
1. Conditioning: a helical blade moves downwards and then upwards in the granular bed to achieve a uniform and reproducible initial state.

2. Compaction: a vented piston is used to consolidate the granular bed at a selected normal load.

3. Pre-shearing: the granular bed is pre-sheared at the same normal load as the compaction step, until a steady state flow is achieved (uniform density and strain independent).

4. Shearing: after pre-shearing, the normal stress is lowered and the granular bed is sheared to obtain a yield point. The process of pre-shearing/shearing is repeated 4 or 5 times at different normal stresses in order to construct the yield locus (see Fig.2b).
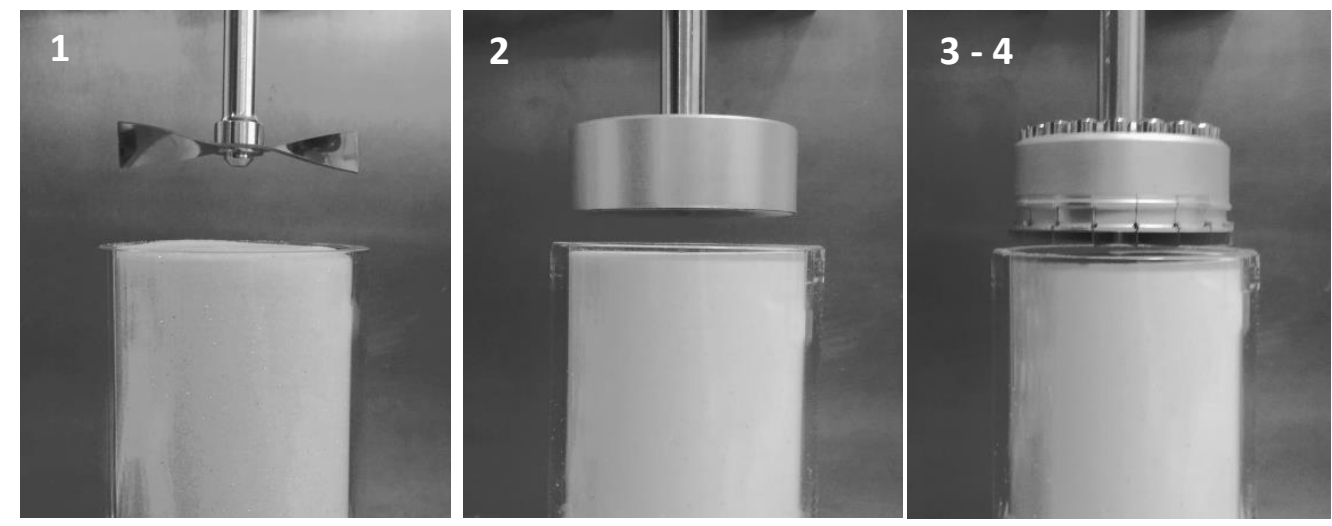

Fig. 1 The experimental operational procedure of an FT4 sheartest : (1) conditioning (2) compaction ( $3-4)$ pre-shearing and shearing.

The FT4 shear tester provides different methods for testing by adjusting the consolidation stress to $3,6,9$ or $15 \mathrm{kPa}$. In this paper, the consolidation stress will be labelled $\sigma_{\mathrm{c}}$, which should not be confused with the Unconfined Yield Strength. The normal stresses used during shearing should be in the range of $20 \%$ to $80 \%$ of the consolidation stress (Schulze, 2008). The influence of the consolidation stress on the experimental shear stress results will be investigated in the present work using DEM, and will be compared with the literature. Unless mentioned otherwise, the shear-test method used in this work consists of applying a normal stress equal to $15 \mathrm{kPa}$ for compaction and pre-shearing. Four normal stresses applied during shearing are in this order $9,8,7$ and $6(\mathrm{kPa})$. A pre-shearing at $15 \mathrm{kPa}$ precedes each shearing step.

The corresponding shear stresses to the varied normal stresses allow to construct the yield locus: $\tau=f(\sigma)$ curve (see Fig. 2b). The slope of the yield locus is the Angle of Internal Friction, $\phi$, and the intercept of the yield locus with the shear stress axis gives the apparent cohesion, $C$, according to Mohr-Coulomb's equation:

$\tau=\sigma \tan (\Phi)+C$. 

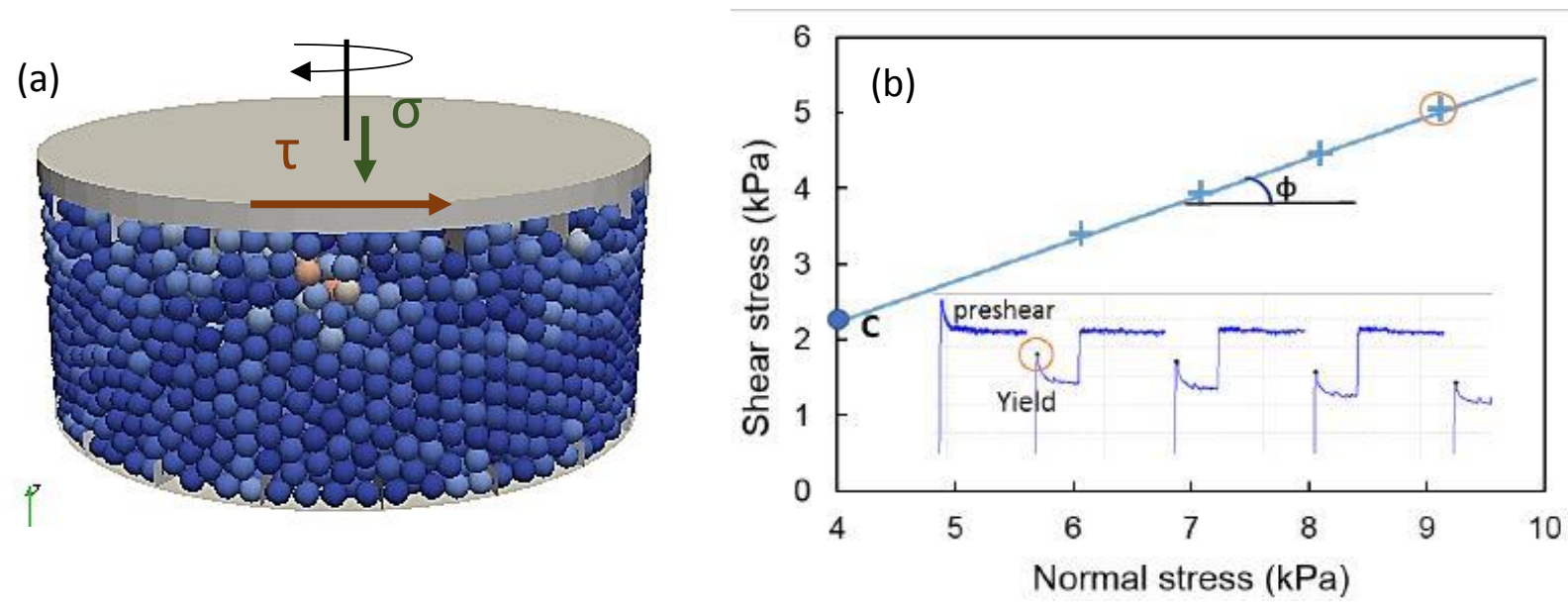

Fig. 2 (a) Snapshot of FT4 shear cell from DEM simulation. (b) Typical shear-test result showing the shear stress responses during pre-shearing and shearing and the obtained yield locus, which gives the angle of internal friction, $\phi$, and apparent cohesion, $C$.

\subsection{Discrete Element Method (DEM)}

The governing equations of the particle motion in DEM are based on Newton's second law. The translational and rotational coordinates of each particle, $i$, are given by integrating the following equations:

$m_{i} \frac{d^{2} x_{i}}{d t^{2}}=\sum_{j}\left(F_{n}+F_{t}\right)+m_{i} g$,

$I_{i} \frac{d \omega_{i}}{d t}=\sum_{j}\left(R_{i} \cdot F_{t}\right)+\tau_{i j}$

where $x_{i}, m_{i}, R_{i} I_{i}$ and $\omega_{i}$ are the position, mass, radius, moment of inertia, and angular velocity of particle $i$. $\tau_{i j}$ is the rolling resistance torque of particle $i$ in contact with particle $j . F_{n}$ and $F_{t}$ are the normal and tangential components of the contact force as a result of the interactions with the neighbours particles. These forces of contact are estimated by allowing the particles to slightly interpenetrate each other as a way to simplify the real deformation of materials (Cundall and Strack, 1979). In this work, the Hertz-Mindlin \& Deresiewicz models were used to compute the contact forces

$F_{n}=k_{n} \delta_{n}^{3 / 2}+D_{n} \dot{\delta_{n}}($ Hertz, 1896)

$F_{t}=\min \left[\left(k_{t} \delta_{t}+D_{t} \dot{\delta}_{t}\right), \mu F_{n}\right]$ (Mindlin \& Deresiewicz, 1953)

where $k_{n}$ and $k_{t}$ are the normal and tangential stiffness coefficients respectively. $D_{n}$ and $D_{t}$ are the normal and tangential damping coefficients respectively. $\delta_{n}$ and $\delta_{t}$ are the normal and tangential interpenetration distances between particles $i$ and $j$ and $\dot{\delta}_{n}$ and $\dot{\delta}_{t}$ are the relative normal and tangential velocities. The expressions of the stiffness and damping coefficients are reported in details in (Tsuji et al., 1992) (Louati et al., 2016). The tangential force (Eq. 5) takes the minimum value of the Mindlin and Deresiewicz or the Coulomb's law of friction expressed as $\mu F_{n}$, where $\mu$ is the sliding friction coefficient.

The JKR model was used to compute the adhesive forces between particles (Johnson et al., 1971) (Kodam et al., 2009) (Kempton et al., 2012). A cohesive term is added to the normal contact force through a parameter, $\gamma\left(\mathrm{J} . \mathrm{m}^{-2}\right)$, not necessarily representative of the surface tension (i.e. $\gamma$ may take high values in order to obtain a significant effect of the cohesive forces, especially for system of large particulate sizes where the gravitational forces are dominant). 
$F_{n}^{\prime}=F_{n}-\sqrt{4 F_{n} F_{c}}$

$F_{c}=1.5 \pi \gamma R_{i j}^{*} \quad\left(\gamma=\gamma_{i}+\gamma_{j}\right)$

where $F_{n}^{\prime}$ is the normal contact force including the cohesive force denoted by $F_{c}$ and $\gamma$ is the surface energy $\left(\mathrm{J} . \mathrm{m}^{-2}\right) \cdot R_{i j}^{*}$ is the equivalent radius of the two spheres in contact $i$ and $j$ and it is calculated as:

$\frac{1}{R_{i j}^{*}}=\frac{1}{R_{i}}+\frac{1}{R_{j}}$

The interparticle forces acting at the contact between particles or a particle and a wall produces a torque that leads to the rotation of particles. The rolling resistance is a torque that opposes to that rotational motion. Different mechanisms and particle properties can contribute to the rolling resistance including: micro-slipping (if the bodies in contact have dissimilar elastic constants), plastic deformation, viscoelastic deformation, surface adhesion, shape of particle, etc. (Ai et al., 2011). In DEM simulation, the rolling resistance is controlled by the implementation of a rolling friction model that adds an additional torque resistance $\tau_{i j}$ (Zhou et al, 1999). The direction of the torque is against the relative rotation between the bodies in contact:

$\tau_{i j}=-\mu_{R} F_{n} d_{i j} \frac{\omega_{r}}{\left\|\omega_{r}\right\|}$

$d_{i j}=\left\|\vec{O}_{i}-\vec{O}_{j}\right\|$

$\vec{\omega}_{r}=\vec{\omega}_{i}-\vec{\omega}_{j}$

where $\mu_{R}$ is the rolling friction coefficient, $d_{i j}$ is the distance between the two centers $O_{i}$ and $O_{j}$ of the two particles in contact. $\omega_{i}$ and $\omega_{j}$ are the angular velocities of particles $i$ and $j$ respectively and $\omega_{r}$ is the relative angular velocity between them.

\subsection{The numerical FT4 shear test}

The discrete element simulation was made using LIGGGHTS $^{\circledR}$, which is an open-source software based on the molecular dynamics code LAMMPS (Kloss and Goniva, 2011). The simulation of the shear test was performed in a similar way to the experimental method as described in section 2.1. However, parts of the operational experimental procedure appeared to be not necessary. In fact, the spheres are generated and settled down by free fall on the shear cell and only the DEM parameters such as sliding friction coefficient, rolling friction, restitution coefficient, density, etc. can influence the powder bed packing, which may affect the results. Therefore, the conditioning step is not simulated. The filling condition such as the granular bed height and the minimum particles number in the shear cell were optimised (Bednarek, 2018) and are reproduced in a similar way for all the shear tests. Moreover, the diameters of the vessel and the lid have been taken identical to avoid the loss of particles in the interstice. This phenomenon is occurred since the Young's modulus has been reduced during filling procedure in order to speed up the simulation time. Then, once the lid is in contact with the powder, the Young's modulus is brought back to its targeted value. At this stage, an interstice between the lid and the vessel wall may lead to loss of particles. 


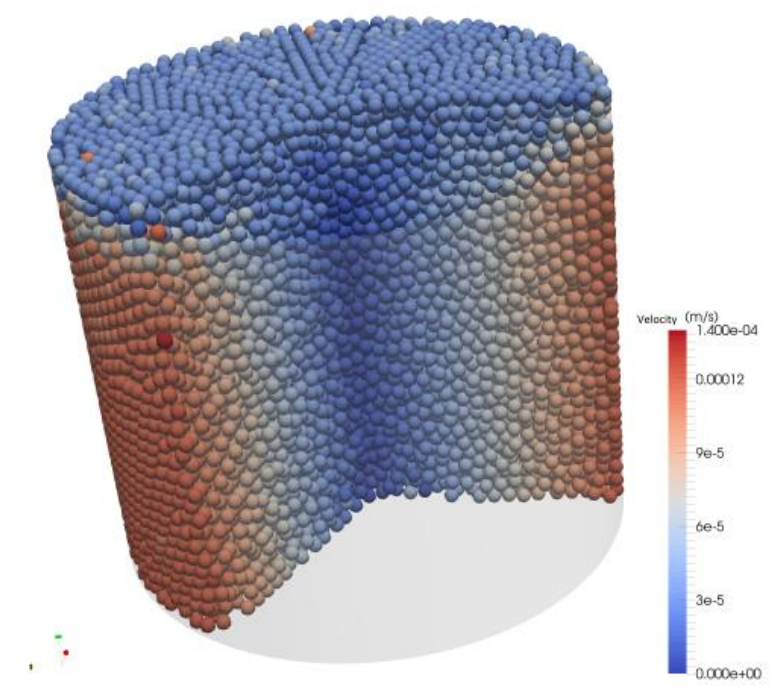

Fig.3. Velocity of particles within a shear cell with height/diameter ratio equal to the original geometry.

The dimensions of the shear cell are defined according to the optimal number of particles of the granular bed. Fig. 3 shows the velocity of particles within a shear cell with the original FT4 geometry. It can be observed that the shearing plan is located at the $3 / 4$ of the height and that the half bottom shows a perfect isotropy (particles move with the wall). Hence, to speed up the simulation time, only a layer of about 10 particles was conserved in this study (see Fig. 2a). The cell diameter is about 25.5 particles side by side. This is the minimum numbers which leads to particles smaller than the space between the paddles of the lid. Moreover, previous study from our lab (Bednarek et al., 2017) showed that a higher number of particles per diameter does not lead to very different results.

After the particles were settled down, a normal load is applied through the lid and maintained by servocontrol during pre-compaction, pre-shearing and shearing steps. For the experimental device, the vessel containing the powder is motionless and the lid is rotating to provoke the shearing. In the simulation, the vessel is rotating but the lid is not, which allows better servo-control of the applied normal stress. This last point comes from some limitation of LIGGGHTS. It is, indeed, not possible to control the vertical motion of a mesh while it is rotating. We assume that this has a very little effect on the results since the rotation speed is very low.

Different assumptions can be stated to calculate the shear stress. Since the further objective of this work is to meet the experimental conditions, it was decided to use the same equation implemented in the real FT4, assuming, as did (Carr and Walker, 1968), that the shear stress is homogeneous. The shear stress is calculated from the torque $\Gamma$ as follow

$\tau=\frac{3}{2 \pi R^{3}} \Gamma$

The rotational velocity is set to $0.05 \mathrm{rpm}$ allowing a slow frictional regime, where the shear stress is independent of the shear rate (Tardos et al., 2003). The input parameters of the material properties are given in table. 1. It is worth to mention that a monosize spheres of $3 \mathrm{~mm}$ in diameter are considered here, which may lead to unrealistic behaviour. Indeed, the results of this study are intended to be used to calibrate particle parameters of monodisperse spheres. Polydispersity may lead to largely increase the computational time for systems with large particles number. The particle rolling friction coefficient was set to 0.01 . The 
particle-particle sliding friction coefficient and the particle surface energy are the parameters to be varied in the simulations in order to quantify their influence on the angle of internal friction and the bulk cohesion. The surface energy between the particle and the wall of the shear cell was set to zero (no cohesion but friction). In this work, we focus on the properties of the granular material. Nevertheless, the effect of the wall properties could be significant and we encourage to investigate these parameters. It has been reported in a recent study about the dynamics of granular flow in an annular shear cell using DEM that the particlewall friction coefficient affects the shear localisation (Artoni et al, 2018).

Table 1. Simulation parameters for the FT4 shear test.

\begin{tabular}{lcc}
\hline Parameters & $\begin{array}{c}\text { Particle } \\
\text { * p-p contact }\end{array}$ & $\begin{array}{c}\text { Wall } \\
\text { * p-w contact }\end{array}$ \\
\hline $\mathrm{R}(\mathrm{mm})$ & 1.5 & - \\
Density $\left(\mathrm{kg} \cdot \mathrm{m}^{-3}\right)$ & 2500 & - \\
Young's modulus (MPa) & 10 & 1000 \\
Poisson's ratio & 0.25 & 0.25 \\
Coefficient of restitution & $0.3^{*}$ & $0.3^{*}$ \\
Rolling friction coefficient & $0.01^{*}$ & $0.01^{*}$ \\
Sliding friction coefficient & to be varied $[0.1-1]^{*}$ & $0.5^{*}$ \\
Surface energy $\left(\mathrm{J} \cdot \mathrm{m}^{-2}\right)$ & to be varied $[0-25]^{*}$ & $0 *$ \\
\hline
\end{tabular}

\section{Results and discussions}

\subsection{Simulation results of the FT4 shear test}

In this section, we investigate the shear test results from DEM simulations. Two granular materials are considered for comparison purpose: $\gamma$ is zero for the noncohesive case and $\gamma=10 \mathrm{~J} . \mathrm{m}-2$ for the cohesive case. The order of magnitude of $\gamma$ was chosen relatively high in order to observe significant cohesive effect on the macroscopic behaviour of granular materials.

Fig.4a shows the shear stress responses during pre-shearing (dotted line) and shearing (solid line) of non-cohesive (blue) and cohesive (magenta) granular materials. $\mathrm{t}^{*}$ is the shearing time relative to the time needed to cross one entire sphere. The circles mark the incipient failure point (peak) and the squares are the average values of the shear stresses at steady state (plateau). The incipient failure point indicates the failure of the granular bed and the start of the shear flow. The steady state is reached when a shear plane is created and the shear stress tends toward a constant value. The peak is clearly observed during pre-shearing on the
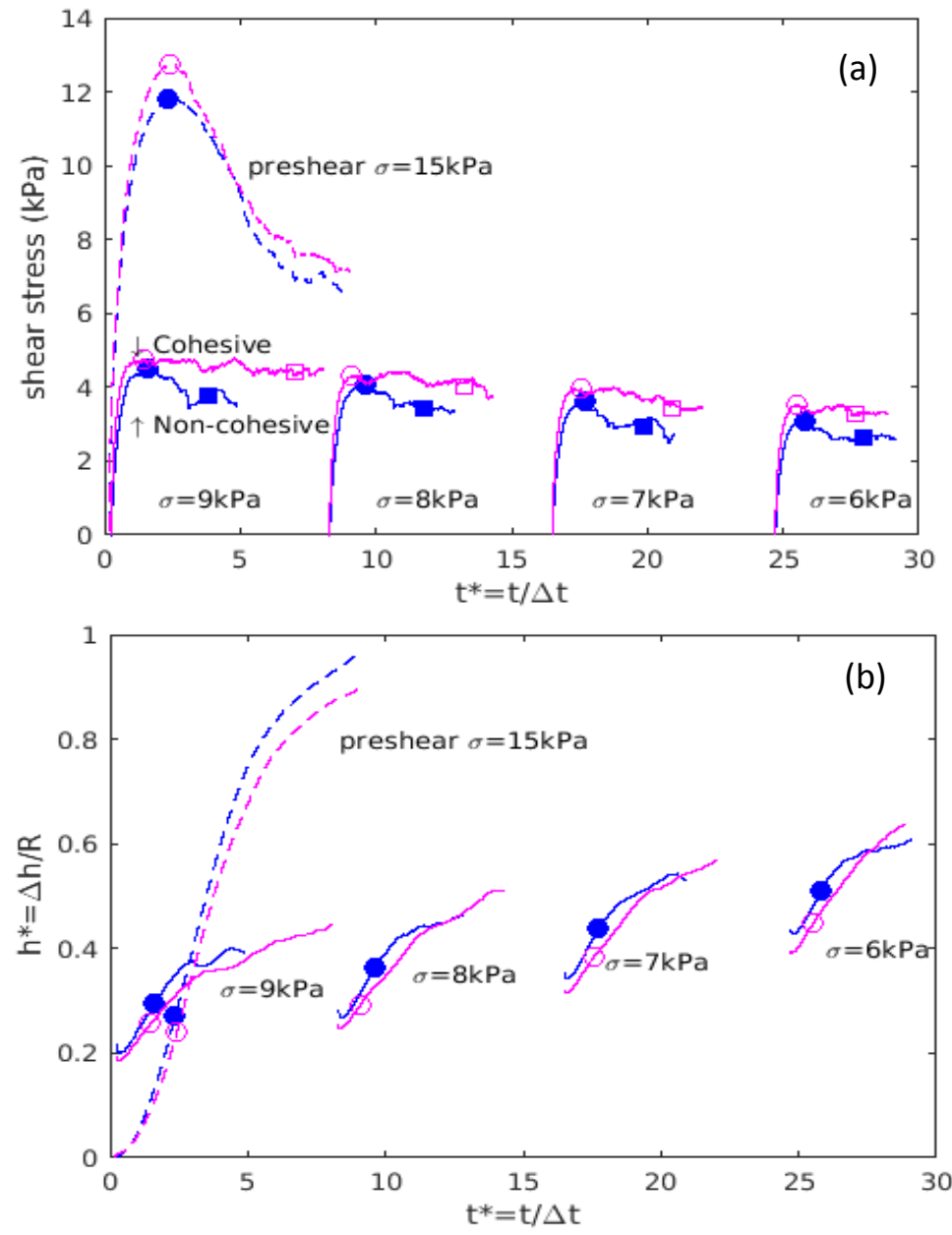
contrary to the shearing. The shear stress increases in the cohesive case because of the adhesive force incremented to the normal contact forces. Therefore the torque required for shearing increases and so the shear stress. However, no peak is observed for the shear stress signal of cohesive granular materials.

The investigation of the dilatancy of the granular bed (Fig.4b), quantified by the dimensionless bed expansion $h^{*}$, shows a dependency on the normal stress applied. At high consolidation during pre-shearing (15 $\mathrm{kPa})$, the incipient failure occurs at about $25 \%$ of the particle radius. After the failure point, the dilatancy, $h^{*}$, continues to increase to reach the equivalent of one particle radius. After pre-shearing, the normal stress is lowered for shearing and the dilatancy of the bed at the incipient failure occurs at $10 \%$ of the particle radius and increases up to about $25 \%$.

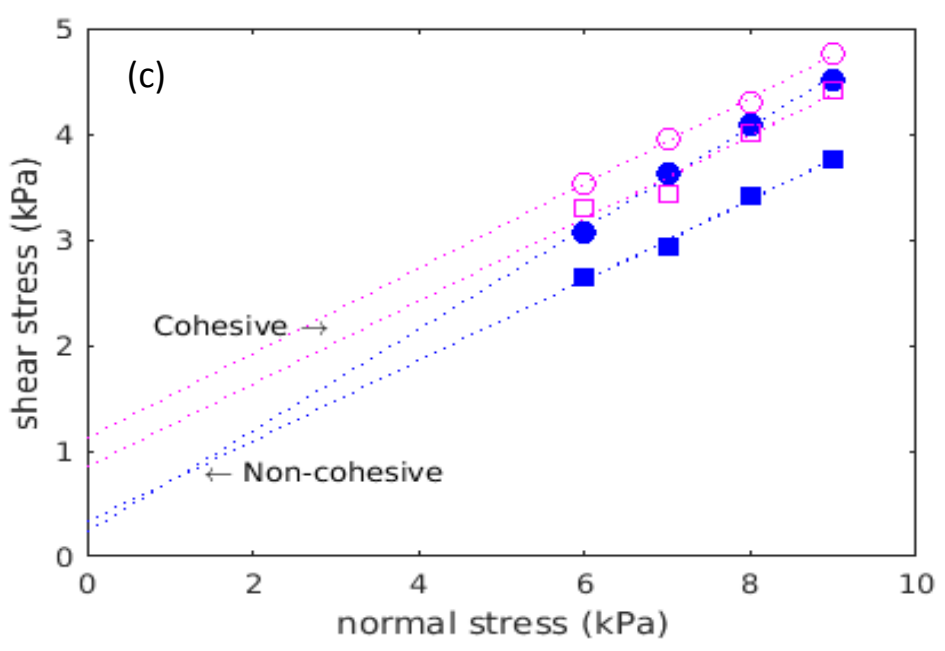

Fig. 4 (a) shear stress response during pre-shearing /shearing of non-cohesive (blue) and cohesive (magenta) granular material. (b) Dilation of the granular bed during pre-shearing and shearing. (c) The yield loci (circles) and the steady-state data (squares).

Indeed, the normal stress is controlled by controlling the position of the lid, which moves upwards in order to decrease the normal stress and viceversa. Thus, lower consolidation state of the granular bed occurs after decreasing the normal stress for shearing. This leads to loose configuration of the granular material allowing an easier rearrangement of the particles. Therefore, a smaller dilatancy of the bed is required to initiate shearing. Consequently, the shear stress at the incipient failure point is decreased.

The appearance of a peak on the shear test results from DEM simulation is also influenced by the input parameters of the particle properties such as the Young's modulus and the sliding friction coefficient. Generally, a decrease of the Young's modulus in order to gain on the computational time leads to decrease the shear stress magnitude at the peak and not at the steady state (Louati, 2016; Bednarek, 2018). Simons et al., (2015) has reported a decrease of the shear stress magnitude even at steady state. Moreover, the dilatancy takes longer time for smaller value of the Young's modulus since particles are softer. Besides, the sliding friction coefficient influences the shear stress response: the increase of the sliding friction coefficient leads to an increase of the shear stress. However, it influences a very little the moment when the peak occurs, as shown on Fig.5. 


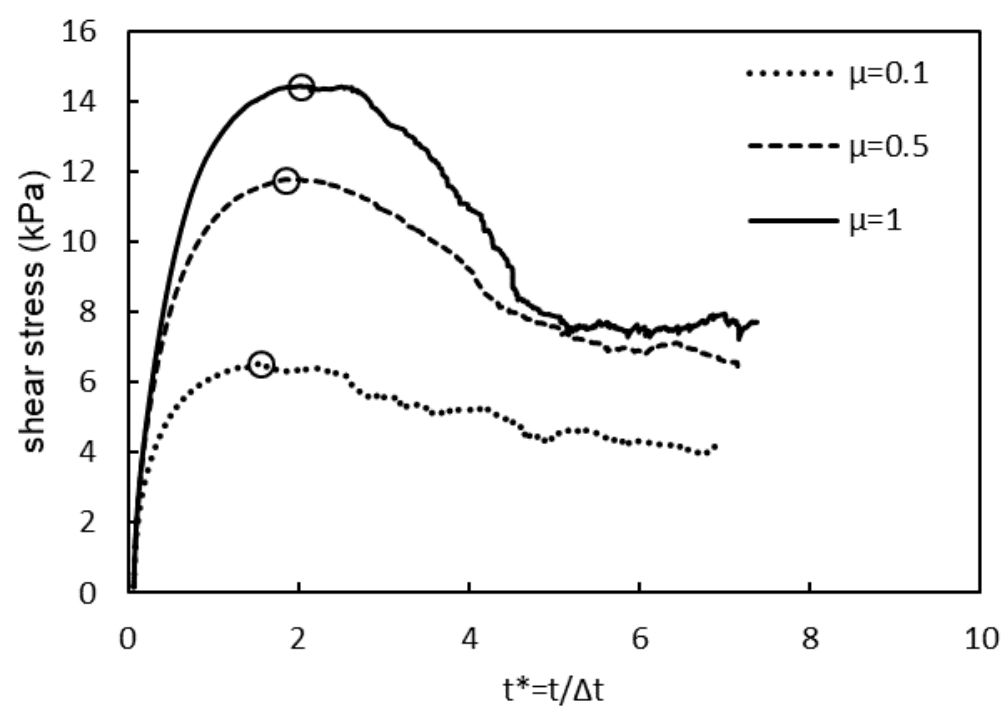

Fig.5. Influence of the sliding friction coefficient on the moment when the peak occurs (pre-shearing step)

The surface energy $\gamma$, which induces cohesion between particles, also affects the incipient failure point. Fig.4a shows that for cohesive granular materials, there is no sharp peak but rather a smooth maximum point marked by a circle for cohesive granular materials. The dilatancy seems to increase further compared to the non-cohesive granular material. Indeed, cohesive granular materials are more compressible and hence the dilation occurred during shearing takes a little longer.

Fig.4c shows the yield loci computed with the incipient failure values of the shear stress (circles) and the steady state stress values (squares). The shear stress at steady state is an average value of the stresses at the plateau. The angle of internal friction can be obtained by linear regression of the yield locus, denoted by $\phi_{\text {if }}$, and describes the maximum bulk friction during incipient failure at a given consolidation stress. It can also be determined from the shear stress response at steady state, denoted by $\phi_{s s}$, and describes the granular flow behaviour. The bulk cohesion is obtained by extrapolation of the yield locus and corresponds to the intercept with the shear stress axis at zero normal stress. For cohesive granular material, the yield locus is non-linear: the slope decreases by increasing the normal stress (Louati et al, 2015). From Fig.4a and 4.c, we can see that both the yield loci and the values at steady state permit to distinguish non-cohesive from cohesive flow; the shear resistance is higher for cohesive granular material. Table. 2 summarises the values of $\phi_{\text {if }}, \phi_{\mathrm{ss}}$ and $\mathrm{C}$ for non-cohesive and cohesive materials. Indeed, the values of $\phi_{\mathrm{if}}$ and $\phi_{\mathrm{ss}}$ for cohesive materials are very close in comparison to the non-cohesive case. $\phi_{\text {if }}$ slightly decreases for cohesive granular material while $\phi_{s s}$ has almost the same value for both cases. Moreover, the difference between the non-cohesive and cohesive steady state shear stresses (squares) is larger than the difference between stresses at the incipient failure (circles). This leads to two comments: first, if the peak was not observed for cohesive materials then it is not appropriate to compare the incipient failure point? Second, it joins the idea that the interparticle cohesion contributes more at the steady state flow than at the incipient failure (Shi et al, 2018). 
Table. 2 Comparison of the angle of internal friction at the incipient failure $\phi_{\text {if }}$ and at steady state $\phi_{\mathrm{ss}}, \Delta \phi=\phi_{\mathrm{if}}-\phi_{\mathrm{ss}}$ and the apparent cohesion C for noncohesive and cohesive granular materials.

\begin{tabular}{lcc}
\hline Macroscopic properties & $\begin{array}{c}\text { Non-cohesive } \\
(\gamma=0)\end{array}$ & $\begin{array}{c}\text { Cohesive } \\
\left(\gamma=10 \mathrm{~J} . \mathrm{m}^{-2}\right)\end{array}$ \\
\hline $\mathrm{C}(\mathrm{Pa})$ & 240 & 1120 \\
$\phi_{\mathrm{if}}\left(\left(^{\circ}\right)\right.$ & 25.5 & 21.9 \\
$\phi_{\mathrm{ss}}\left({ }^{\circ}\right)$ & 20.9 & 21.4 \\
$\Delta \phi\left(^{\circ}\right)$ & 4.6 & 0.5 \\
\hline
\end{tabular}

\subsection{Influence of consolidation on the shear test results}

Depending on the measurement method used in the FT4 shear test, the experimental results could be deviated (Vasilenko et al., 2013; koynov et al., 2015; Shi et al., 2018). In this section, we investigate the effect of the consolidation on the shear stress response obtained from DEM simulations and we compare the results with the literature (experimental results).

The shear test procedure is the same as described in section 2.1. The only difference is the consolidation stress (during pre-shearing) and the normal stresses during shearing. Only the non-cohesive case is illustrated in Fig. 6 for sake of clarity. Table. 3 summarises the three test methods that have been carried out.

Table.3 Three shear test methods to study the effect of the consolidation stress.

\begin{tabular}{lll}
\hline Tests & $\boldsymbol{\sigma}_{\mathrm{c}}(\mathbf{k P a})$ & $\boldsymbol{\sigma}(\mathbf{k P a})$ \\
\hline 1 (blue) & 15 & $9,8,7,6$ \\
2 (red) & 9 & $7,6,5,4$ \\
3 (green) & 6 & $4,3.5,3,2.5$ \\
\hline
\end{tabular}

As it can be observed in Fig.6a, the intensity of the shear stress peak is dependent on the normal stress applied. The larger is the normal stress, the higher is the shear stress peak value relative to the steady state value. This is explained by the fact that the number of contacts between particles increases with
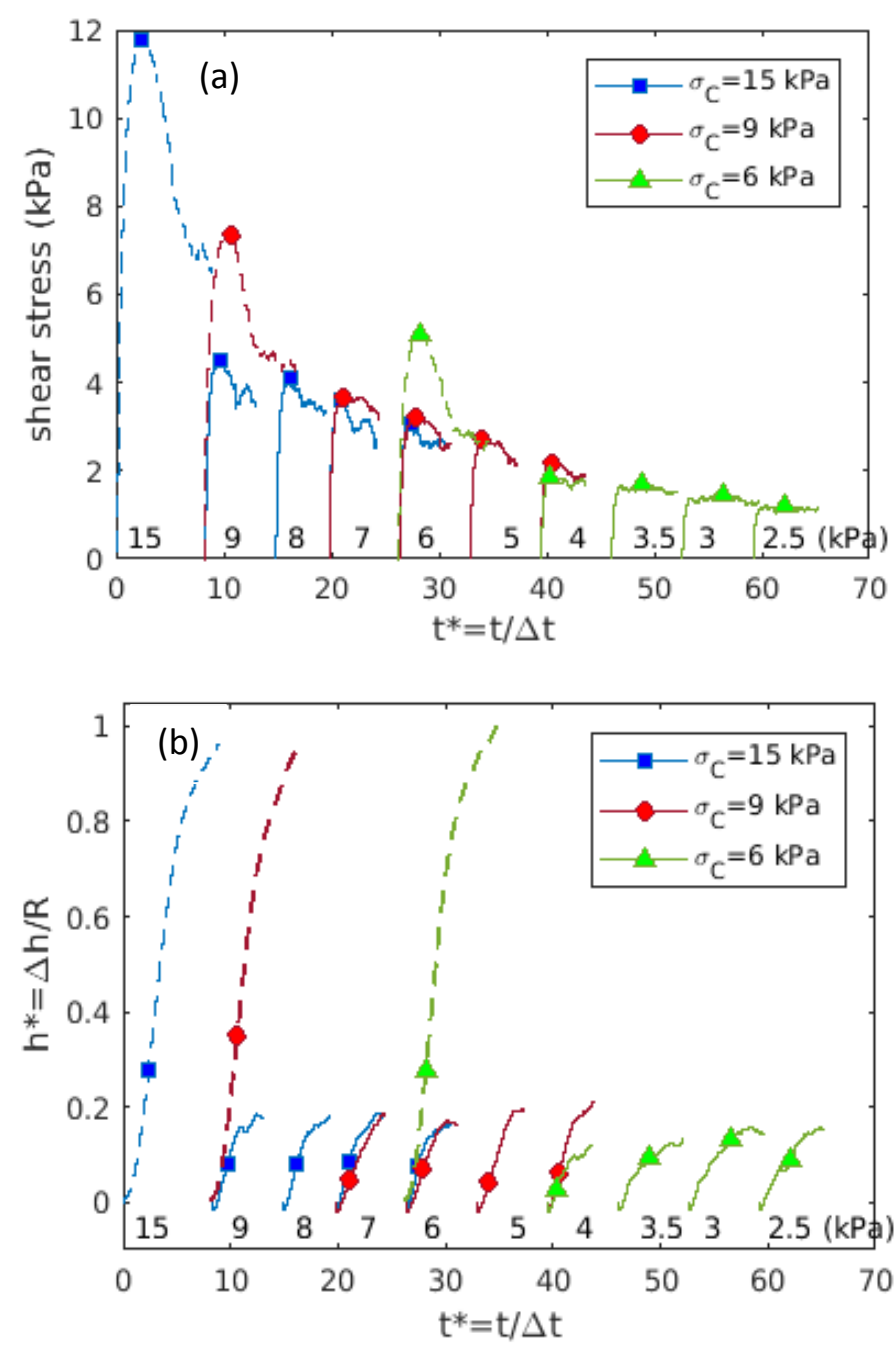

Fig.6. Influence of the consolidation stress on the shear stress response (a) and the corresponding dilatancy (b) of non-cohesive granular bed. 
loading and therefore the resistance of the granular materials to shearing.

Fig.6b shows that the dilatancy is actually not affected by the normal stress loading. After compaction, the pre-shearing leads to a dilatancy equivalent to the size of a particle radius regardless the consolidation stress (15, 9 or $6 \mathrm{kPa}$ ). During shearing, dilatancy is about $15-20 \%$ of the particle radius. However, for low consolidation stress $\left(\sigma_{\mathrm{c}}=6 \mathrm{kPa}\right)$, the dilatancy of the granular bed seems to be more sensitive to the normal stress; the dilatancy increases with the normal stress decreasing. A threshold is maybe reached between 6 and $9 \mathrm{kPa}$, where there is no dependency on the consolidation stress beyond that value.

A quantitative investigation of the effect of consolidation stress on the angle of internal friction and apparent cohesion for noncohesive $(\gamma=0)$, slightly cohesive $\left(\gamma=1 \mathrm{~J} \cdot \mathrm{m}^{-2}\right)$ and very cohesive granular materials $(\gamma=10$ J. $\mathrm{m}^{-2}$ ) is given in Fig.7. Regarding the angle of internal friction (Fig.7a), it is observed a decrease of $\phi$ for an increase in the consolidation stress for the cohesive case (red and black). No clear dependency of $\phi$ on the consolidation stress is observed for the noncohesive case (blue) if we take into consideration the large standard deviation of $\phi$ at $6 \mathrm{kPa}$. Shi et al. (2018), have reported similar variations of $\phi_{\mathrm{ss}}$ (from steady-state flow) and independency of $\phi$ (from incipient failure) on the consolidation stress.

These experimental observations were made on a Schulze ring shear tester, for consolidation stresses of 5, 20 and $35 \mathrm{kPa}$ and for various range of particle sizes (from $\sim 1$ and up to $\sim 1000 \mu \mathrm{m}$ ). Regarding the apparent cohesion, it has been reported an increase of $C$ with the normal stress increasing, more pronounced for cohesive granular material. Here, the cohesion slightly increases with the consolidation stress.

\subsection{Influence of microscopic coefficients $\mu$ and $\gamma$ on macroscopic properties $\phi$ and $C$.}

In this section, a number of shear-test measurements were performed for different sliding friction coefficients and surface energies in order to provide a quantitative analysis of the angle of internal friction and the apparent cohesion. It is worthwhile to mention that quantitative DEM shear test results are sensitive to many parameters including the particle size, particle size distribution, particle stiffness, granular packing and interparticle interaction parameters such as friction, cohesion, etc. Therefore, the quantitative data and correlations may change for other particulate systems. 


\section{Sliding friction coefficient $\mu$}

Previous works have studied the effect of the particle sliding friction on the shear stress response and the angle of internal friction using DEM simulation of direct shear tests (Coetzee et al., 2017; Louati et al., 2016; Simons et al., 2015; Coetzee and Els, 2009). It was reported that the shear stress and the angle of internal friction increase non-linearly with the sliding friction coefficient and then reach an asymptotic value. In this work, we investigate the variation of $\phi$ with $\mu$ obtained from DEM simulations of FT4 shear tester, which has no record in the literature. Furthermore, we investigate the effect of the surface energy on the angle of internal friction. Fig. 8 shows that the angle of internal friction increases with the friction coefficient in the range of 0.1 to 1 with an asymptotic value observed around $\mu \sim 0.5$. The values of $\phi$ are in the range of $15^{\circ}$ to $35^{\circ}$ and are expected to be smaller than the experimental measurement because of the effect of particles angularity. In other words, if a geometric parameter for the particle shapes is considered in the DEM simulation, $\phi$ is expected to increase (Zhao, et al, 2015). The particles used in the simulation are perfectly spherical, which decreases the value of $\phi$ (Coetzee, 2016). The surface energy slightly affects the values of the angle of internal friction (see Fig.8). Thus, one correlation is obtained for different values of $\gamma$.

$\phi_{\text {iff }}\left({ }^{\circ}\right) \sim 6.5 \ln (\mu)+32(\mathrm{e} \sim 14 \%), \quad$ for $0.1 \leq \mu \leq 1$ and $0 \leq \gamma \leq 25$

\section{Surface energy $\gamma$}

There is a lack of quantitative studies of the apparent cohesion measured experimentally or numerically from shear tests in the literature. As mentioned before, the apparent cohesion is obtained by linear regression of the yield locus and extrapolation at zero normal stress (see Eq.1). However, the yield locus tends to curve downwards at low normal stresses, particularly for cohesive granular materials so the evaluation of the cohesion is overestimated (Schulze, 2008; Vasilenko et al., 2013, Louati et al., 2017). Besides, Shi et al. have compared the experimental apparent cohesion obtained from different devices [Jenike, Schulze ring shear cell, FT4, etc.] and reported discrepancies between the results (Shi et al., 2018). In particular, the FT4 shear test gives a relatively lower value of the cohesion compared to other devices. Furthermore, the repeatability of the measurement procedure for the same device leads to a certain deviation even for the FT4, where the measurement operations are completely automated (Shi et al., 2018). The cohesion measured by FT4 can be sensitive to the consolidation stresses especially for compressible (cohesive) powders (Vasilenko et al., 2011). In this work of DEM simulations of the FT4 shear tests, we report that results are reproducible if the vessel is filled in the same way, i.e. the rearrangement of the granular bed is not affected during filling. The analysis of the yield locus for a large range of normal stresses shows that it tends to curve downwards for cohesive granular materials when $\sigma$ tends to zero. The effect of the consolidation stress was discussed in section 3.2. Fig. 9 shows the variation of the apparent cohesion with the surface energy, where $C$ increases linearly with $\gamma$. However, the standard deviation calculated on the linear regression of yield loci are significant, especially when the sliding friction coefficient is larger than 0.5. It is complicated to obtain a good $C(\gamma)$ correlation for $\mu$ in the range of 0.1 to 1 with these scattered data. However, for $\mu$ in the range of 0.25 to 0.5 , the standard deviation of the data are very acceptable and the curve $C(\gamma)$ have the same tendency (see Fig. 9). A relationship to estimate the cohesion from the surface energy is given as:

$\mathrm{C}(\mathrm{Pa}) \sim 90 \gamma+120, \quad$ for $0.25 \leq \mu \leq 0.5$

Eq. 13 and Eq. 14 can be useful for calibration of particle properties from macroscopic measurement of the angle of internal friction and the apparent cohesion $\mathrm{C}$. It is worth mentioning that these two correlations 
result from simulations of monodisperse particles. The effect of the particle size distributions as well as other parameters such as the rolling friction may lead to different correlations.

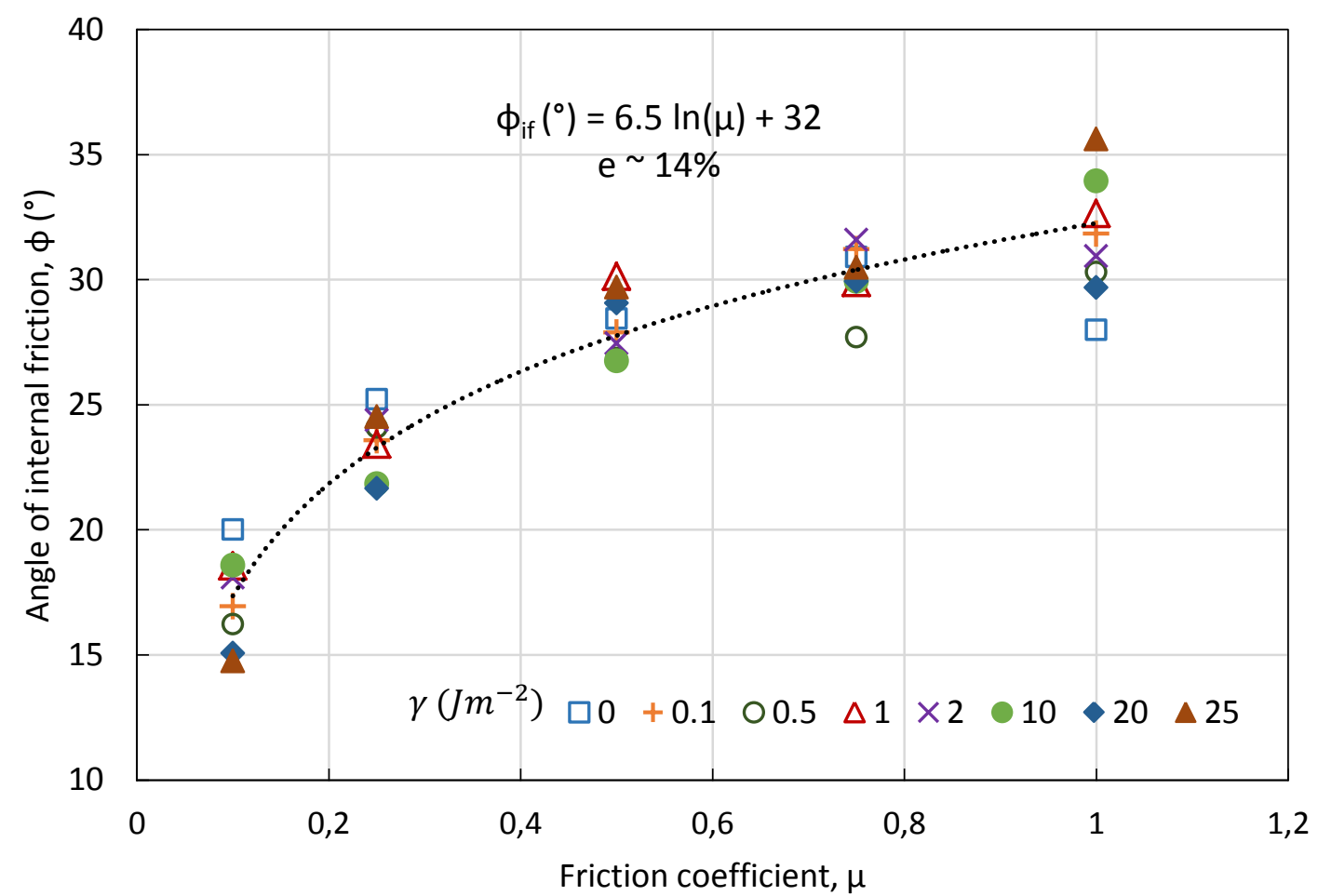

Fig.8. Variation of the angle of internal friction as a function of the frictrion coefficient $\mu$ for different surface energy values. 


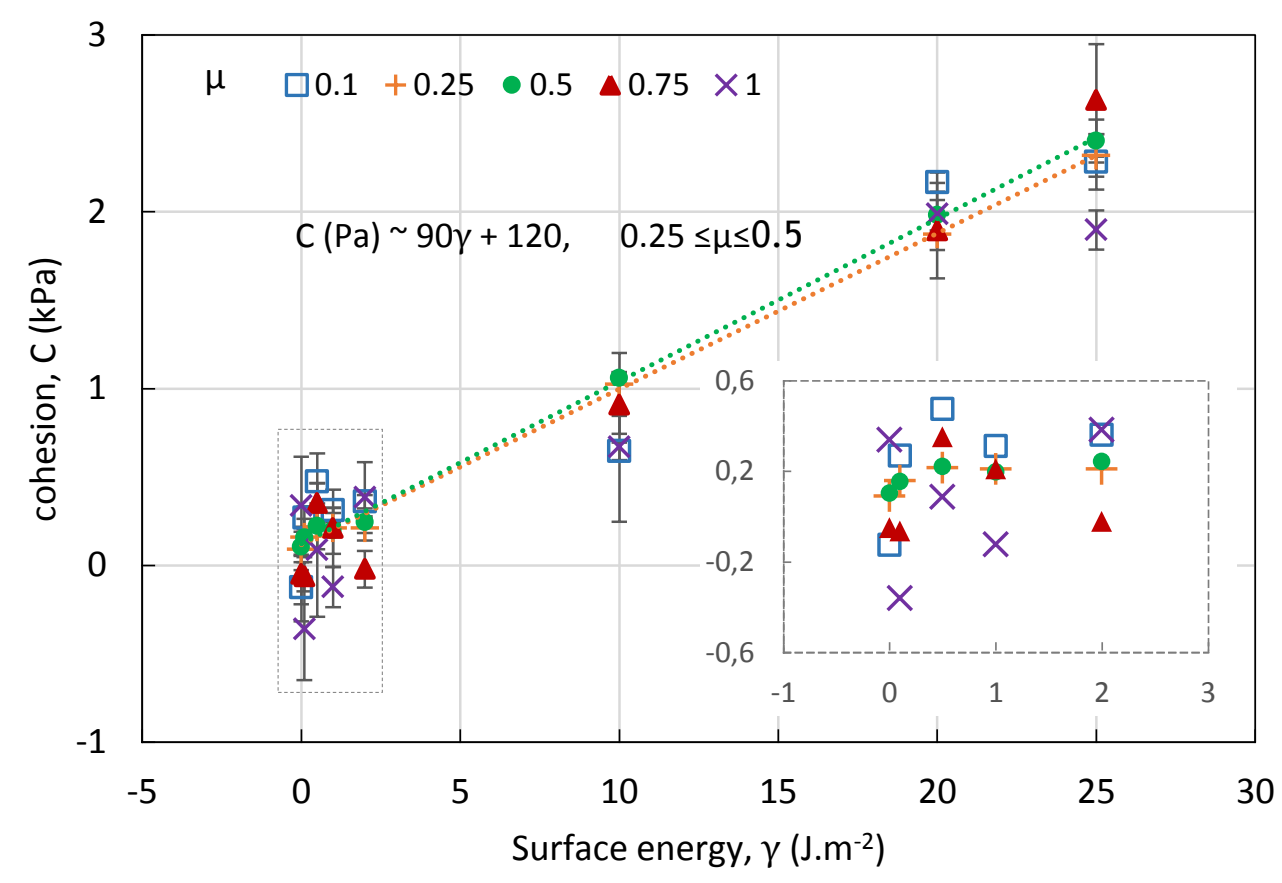

Fig.9. Variation of the apparent cohesion as a function of the surface energy for granular materials with different friction coefficients.

\section{Conclusions}

This study gives qualitative and quantitative investigation of the FT4 shear test for cohesive granular material. We first reported that the appearance of the incipient failure peak and the dilatancy of the granular bed are influenced by the particle properties, interparticle interactions such as the surface energy and also the normal stress. Both, the values of the incipient failure (peak) and the steady state shear stress allow to distinguish between cohesive and non-cohesive granular material. Then, we demonstrated that the consolidation stress does not have a significant influence on the dilatancy of the granular bed. However, it affects the magnitude of the apparent cohesion and the angle of internal friction for cohesive granular materials. Finally, two correlations have been established, between the angle of internal friction and the sliding friction coefficient as well as between the cohesion and the surface energy. These two correlations can be useful for DEM calibration of the particle properties to be used for some processes, where the calibration is difficult.

\section{Acknowledgements}

The authors would like to acknowledge Orano Melox for the financial support and permission to publish this paper. 


\section{Notations}

C

Cohesion ( $\mathrm{Pa})$

$D_{n} \quad$ Normal damping coefficient

$D_{t} \quad$ Tangential damping coefficient

e $\quad$ Error (\%)

$\mathrm{F}_{\mathrm{C}} \quad$ Cohesive force $(\mathrm{N})$

$F_{n} \quad$ Normal force (N)

$\mathrm{F}_{\mathrm{t}} \quad$ Tangential force (N)

g Gravitational acceleration $\left(\mathrm{m} / \mathrm{s}^{2}\right)$

$\mathrm{i}, \mathrm{j} \quad$ Particles ID

I

Moment of inertia

$\mathrm{K}_{\mathrm{n}} \quad$ Normal stiffness coefficient

$\mathrm{K}_{\mathrm{t}} \quad$ Tangential stiffness coefficient

$\mathrm{m} \quad$ Mass of particle $(\mathrm{kg})$

PSD Particle size distribution

$\mathrm{R} \quad$ Particle's radius $(\mathrm{m})$

$\mathrm{t} \quad$ Time $(\mathrm{s})$

$t^{*} \quad$ Time relative to the time required by the lid to cross one particle

$x \quad$ Position of particle $(m)$

$\mathrm{d}_{\mathrm{ij}} \quad$ Distance between the centers of two particles $(\mathrm{m})$

$h \quad$ Height of the granular bed $(m)$

$h^{*} \quad$ Height of the bed relative to one particle radius

\section{Greek letters}

$\begin{array}{ll}\gamma & \text { Surface energy }\left(\mathrm{J} / \mathrm{m}^{2}\right) \\ \delta & \text { Overlapping distance }(\mathrm{m}) \\ \mu & \text { Sliding friction coefficient } \\ \mu_{\mathrm{R}} & \text { Rolling friction coefficient } \\ \rho & \text { Density }\left(\mathrm{kg} / \mathrm{m}^{3}\right) \\ \sigma & \text { Normal stress }(\mathrm{Pa}) \\ \sigma_{\mathrm{C}} & \text { Consolidation stress }(\mathrm{Pa}) \\ \phi & \text { Angle of internal friction }\left(^{\circ}\right) \\ \tau & \text { Shear stress }(\mathrm{Pa}) \\ \tau_{\mathrm{ij}} & \text { Torque }(\mathrm{Nm}) \\ \phi & \text { Angle of internal friction }\left({ }^{\circ}\right) \\ \phi_{\mathrm{if}} & \text { Angle of internal friction at the incipient failure }\left(^{\circ}\right) \\ \phi_{\mathrm{ss}} & \text { Angle of internal friction at steady state }\left({ }^{\circ}\right) \\ \omega_{\mathrm{r}} & \text { Relative angular velocity }\end{array}$




\section{References}

Ai J., Chen J.-F., Rotter J.M., Ooi J.Y., Assessment of rolling resistance models in discrete element simulations, Powder Technol., 206 (2011) 269-282.

Artoni R., Soligo A., Paul J.M., Richard P., Shear localization and wall friction in confined dense granular flows, Journal of Fluid Mechanics, 849 (2018) 395-418

ASTM D7891-15, Standard Test Method for Shear Testing of Powders Using the Freeman Technology FT4 Powder Rheometer Shear Cell, ASTM International, West Conshohocken, PA, 2015, www.astm.org

Bednarek X., Martin S., Ndiaye A., Peres V., Bonnefoy O., Calibration of DEM parameters on shear test using Kriging method, EPJ Web of Conf., 140 (2017) 15016.

Bednarek X., Simulation numérique discrète de I'homogénéisation de poudres dans un mélangeur conique à vis, Ph.D. thesis, Ecole des Mines de Saint-Etienne, [confidential] 2018.

Berry R.J., Bradley M.S.A., McGregor R.G., Brookfield powder flow tester-results of round robin tests with CRM-116 limestone powder, Proceedings of the Institution of Mechanical Engineers, Part E: Journal of Process Mechanical Engineering, 229 (2015) 215-230.

Capone G., d'Agostino V., della Valle S., Guida D., Stick-slip instability analysis, Meccanica, 27 (1992) 111-118.

Coetzee C.J., Calibration of the discrete element method and the effect of particle shape, Powder Technol., 297 (2016) 50-70.

Coetzee C.J., Review: Calibration of the discrete element method, Powder Technol., 310 (2017) 104-142.

Coetzee C.J., Els D.N.J., Calibration of granular material parameters for DEM modelling and numerical verification by blade-granular material interaction, Journal of Terramechanics, 46 (2009) 15-26.

Carr J.F., Walker D.M., an annular shear cell for granular material, Powder Technol., 1 (1968), 369-373.

Cundall P.A., Strack O.D.L., A discrete numerical model for granular assemblies, Geotechnique 29 (1979) 47-65.

Freeman R., Measuring the flow properties of consolidated, conditioned and aerated powders - A comparative study using a powder rheometer and a rotational shear cell. Powder Technol., 174 (2007) 25-33.

Gröger T., Katterfeld A., On the numerical calibration of discrete element models for the simulation of bulk solids, Comput. Aided Cem. Eng., 21 (2006) 533-538.

Hertz H., on the contact of elastic solids, J. Reine Angew. Math., 92 (1881) 156-171. Translated and reprinted in English in Hertz's Miscellaneous Papers, Macmillan \& Co., London (1896) Ch. 5.

Jenike A.W., Storage and Flow of Solids, Bulletin No. 123, Bulletin of the University of Utah, 53 (1964).

Jenike A.W., Quantitative design of mass flow bins, Powder Technol., 1 (1967) 237-244.

Johnson K.L., Kendall K., Roberts A.D. Surface energy and the contact of elastic solids, Proc. R. Soc. Lond. A Math. Phys. Sci. 324 (1971) 301-313.

Kempton L., Pinson D., Chew S., Zulli P., Yu A., Simulation of macroscopic deformation using a sub-particle DEM approach, Powder Technol. 223 (2012) 19-26.

Kloss C., Goniva C., Pirker S., LIGGGHTS - open source discrete element of granular materials based on LAMMPS, The Minerals, Metals \& Materials Society (2011) 781-788

Kodam M., Bharadwaj R., Curtis J., Hancock B., Wassgren C., Force model considerations for glued-sphere discrete element method simulations, Chem. Eng. Sci., 64 (2009) 3466-3475.

Koynov S., Glasser B., Muzzio F., Comparison of three rotational shear cell testers: Powder Flowability and bulk density, Powder Technol., 283 (2015) 103-112.

Leturia M., Benali M., Lagarge S., Ronga I., Kashayar S. Characterization of flow properties of cohesive powders: A comparative study of traditional and new testing methods, Powder Technol. 253 (2014) 406-423.

Louati $\mathrm{H}$. Experimental and numerical study of humid granular material: influence of liquid content in quasi-static regime, Ecole Nationale des Mines d'Albi-Carmaux, 2016.

Louati H., Oulahna D., deRyck A., Apparent friction and cohesion of a partially wet granular material in steady-state shear, Powder Technol., 278 (2015) 536-553.

Louati H., Oulahna D., deRyck A., Effect of the particle size and the liquid content on the shear behaviour of wet granular material, Powder Technol., 315 (2017) 398-409.

Mindlin R.D., Deresiewicz H., Elastic spheres in contact under varying oblique force, Trans. ASME, J. Appl. Mech., 20 (1953) 327-344.

Ning Z., Ghadiri M., Distinct element analysis of attrition of granular solids under shear deformation, Chem. Eng. Sci., 61 (2006) $5991-6001$.

Obermayr M., Vrettos C., Eberhard P., Dauwel T., A discrete element model and its experimental validation for the prediction of draft forces in cohesive soil, Journal of Terramechanics, 53 (2014) 93-104.

Robbins M.O., Jamming, friction and unsteady rheology (1999) Cond-mat/9912337.

Schulze D., Powders and bulk solids: behaviour, characterisation, storage and flow, Springer, 2008. 
Schwedes J., Consolidation and flow of cohesive bulk solids, Chemical engineering Science, 57 (2002) 287-294.

Schwedes J., Schulze D., Measurement of flow properties of bulk solids, Powder Technol., 61 (1990) 59-68.

Shi H., Mohanty R., Chakaravarty S., Cabiscol R., Morgeneyer M., Zetzener H., Ooi J.Y., Kwade A., Luding S., Magnanimo V., Effect of Particle Size and Cohesion on Powder Yielding and Flow, KONA Powder and Particle Journal., 35 (2018) 226-250.

Simons T.A.H., Weiler R., Strege S., Bensmann S., Schilling M., Kwade A., A ring shear tester as calibration experiment for DEM simulations in agitated mixers - a sensitivity study, Procedia Engineering., 102 (2015) 741-748.

Tardos G., McNamara S., Talu I., Slow and intermediate flow of a frictional bulk powder in the couette geometry. Powder Technol., 131 (2003) 23-39.

Tsuji Y., Tanaka T., Ishida T., Langrangian numerical simulation of plug flow of cohesionless particles in a horizontal pipe. Powder Technol., 71 (1992) $239-245$.

Vasilenko A., Glasser B.J., Muzzio F.J., Shear and flow behaviour of pharmaceutical blends - Method comparison study, Powder Technol., 208 (2011) 628-639.

Vasilenko A., Koynov S., Glasser B.J., Muzzio F.J., Role of consolidation state on the measurement of bulk density and cohesion, Powder Technol., 239 (2013) 366-373.

Zhao S., Zhou X., Liu W., Discrete element simulations of direct shear tests with particle angularity effect, Granular Matter, 17 (2015) 793-806.

Zhou Y.C., Wright B.D., Yang R.Y., Xu B.H., Yu A.B., Rolling friction in the dynamic simulation of sandpile formation, Physica A: Statistical Mechanics and its Applications, 269 (1999) 536-553. 